Network Working Group

Request for Comments: 1136
S. Hares

D. Katz

Merit/NSFNET

December 1989

Administrative Domains and Routing Domains

A Model for Routing in the Internet

1) Status of this Memo

This RFC proposes a model for describing routing within the Internet. The model is an adaptation of the "OSI Routeing Framework" [1]. This memo does not specify an Internet standard. Comments are welcome. Distribution of this memo is unlimited.

2) Acknowledgement

The authors would like to thank Guy Almes of Rice University for his contributions and insight.

3) Overview

The "core" model of Autonomous Systems [2] formed the basis for the routing model used in the Internet. Due to massive growth and topology changes, the "core" model no longer is in harmony with the reality of today's Internet. Indeed, this situation was foreseen at the outset:

"Ultimately, however, the internet may consist of a number of coequal autonomous systems, any of which may be used...as a transport medium for traffic originating in any system and destined for any system. When this more complex configuration comes into being, it will be inappropriate to regard any one autonomous system as a "core" system" [2].

Furthermore, the Autonomous system concept has been outgrown in certain parts of the Internet, in which the complexity of regional routing has exceeded the limits of the definition of Autonomous systems.

A model which can provide a better match to the Internet can be found in the "OSI Routeing Framework" [1].

This framework proposes a structure of Routing Domains within Administrative Domains. This paper is intended to briefly describe this framework, to outline how this model better fits the reality of 
the present and future Internet, and to show how the model can aid in the construction of well-engineered routing environments.

4) Terminology

The following is a brief glossary of OSI terminology. Formal definitions can be found in the OSI Basic Reference Model [4], the Internal Organization of the Network Layer [5], and the osI Routeing Framework [1].

"Routeing" is the official ISO spelling of what is more commonly spelled "routing." In this paper, the ISO spelling will be used wherever directly quoted from ISO documents, and the common spelling used otherwise.

End System (ES)

An OSI system on which applications run. An End System has full seven-layer OSI functionality. Basically equivalent to an Internet Host.

Intermediate system (IS)

An OSI system that performs routing and relaying functions in order to provide paths between End systems. Intermediate Systems have no functionality above the Network Layer (although a practical realization of an OSI router will have some amount of End System functionality for network management functions, among other things). Basically equivalent to an Internet Router.

Subnetwork (SN)

A communications medium that provides a "direct" path between Network Layer entities. This can be realized via a point-topoint link, a LAN, a Public Data Network, and so forth. This is essentially equivalent to an Internet subnet. It is worth noting that, unlike Internet Subnets, OSI Subnetworks are not necessarily reflected in the addressing hierarchy, so the double meaning of the Internet term "Subnet" (a single IP hop; a part of the address hierarchy) does not hold in the OSI world.

Open Systems Interconnection Environment (OSIE)

The global collection of Open Systems. Basically equivalent to the Internet. 
Network Service Access Point (NSAP)

A conceptual point on the Network/Transport Layer boundary in an End System that is globally addressable (and the address globally unambiguous) in the OSIE. An NSAP represents a service available above the Network Layer (such as a choice of transport protocols). An End System may have multiple NSAPs. An NSAP address is roughly equivalent to the Internet [address, protocol] pair.

Administrative Domain (AD)

"A collection of End Systems, Intermediate systems, and subnetworks operated by a single organization or administrative authority. The components which make up the domain are assumed to interoperate with a significant degree of mutual trust among themselves, but interoperate with other Administrative Domains in a mutually suspicious manner" [1].

A group of hosts, routers, and networks operated and managed by a single organization. Routing within an Administrative Domain is based on a consistent technical plan. An Administrative Domain is viewed from the outside, for purposes of routing, as a cohesive entity, of which the internal structure is unimportant. Information passed by other Administrative Domains is trusted less than information from one's own Administrative Domain.

Administrative Domains can be organized into a loose hierarchy that reflects the availability and authoritativeness of routing information. This hierarchy does not imply administrative containment, nor does it imply a strict tree topology.

Routing Domain (RD)

"A set of End Systems and Intermediate Systems which operate according to the same routeing procedures and which is wholly contained within a single Administrative Domain" [1] .

"A Routeing Domain is a set of ISs and ESs bound by a common routeing procedure; namely:

they are using the same set of routeing metrics, they use compatible metric measurement techniques, they use the same information distribution protocol, and 
they use the same path computation algorithm" [1].

The "OSI Routeing Framework" further provides a formal definition of a Routing Domain, specifying that all ISs within a Routing Domain can determine whether an ES within the domain is reachable, and if so can derive a path to it.

Routing Domains may be divided into subdomains, not unlike subnetting in the Internet. This allows a hierarchical structuring of the domain, permitting containment of the topological details of a subdomain with the resultant reduction in distributed routing information.

An intra-Routing Domain routing protocol is equivalent to an Internet Interior Gateway Protocol (IGP).

An Administrative Domain may contain multiple Routing Domains. A Routing Domain may never span multiple Administrative Domains.

An Administrative Domain may consist of only a single Routing Domain, in which case they are said to be Congruent. A congruent Administrative Domain and Routing Domain is analogous to an Internet Autonomous System.

Common Domain (CD)

"An Administrative Domain which is not a member of a higher level domain. A common domain is the highest level in the routeing hierarchy. There is no single domain above the common domain. In this sense, the routeing hierarchy is in fact multiple hierarchies, with the common domain as the highest element of each hierarchy".

"Where there are multiple common domains, they cooperate as peers to make it possible to route to any NSAP in the OSIE" [1] .

Common Domains have global routing information to the extent necessary to route packets to the proper domain. Each of the several peer national backbones in today's Internet may be considered to be similar to a Common Domain. Note that in the Internet the hierarchical containment implied by the definition of a CD does not really exist; however, there is a level of implicit ordering based on topology and policy issues (the willingness to be used as a transit network) that can be viewed as defining a Common Domain in the Internet. 
For completeness, we offer the following definition for an Internet Autonomous System (AS) :

"An 'autonomous system' consists of a set of gateways, each of which can reach any other gateway in the same system using paths via gateways only in that system. The gateways of a system cooperatively maintain a routing data base using an interior gateway protocol (IGP)..." [3]

5) Environment and Goals

The "OSI Routeing Framework" describes the environment for OSI routing as well as its goals. The environment described is a highly interconnected, highly heterogeneous collection of LANs and public and private networks made up of a diverse collection of equipment from multiple vendors. A number of goals are enumerated, including:

- Support of multiple subnetwork types

- Very large numbers of connected systems

- End System simplicity

- Multiple organizations with mutual distrust and policy/legal restrictions

- High performance

- Robust and dynamic routing in the face of topological changes

The environment and goals described are a good match for those in the Internet. The Internet crosses multiple types of physical media, link layer protocols, and administrative controls. Routers and hosts may come from many vendors. The Internet has become international in scope. Issues of security and the isolation of bad routing information have become international concerns.

The Internet environment, with over 900 highly connected networks (and growing exponentially), is very much like the environment the OSI model aims to describe.

6) Structure of Global Routing

The "OSI Routeing Framework" classifies routing into three types:

- within a Routing Domain

- within an Administrative Domain

- between Administrative Domains

Routing within a Routing Domain involves a high level of mutual trust. This allows the use of complex, tightly-coupled procedures that can make the best use of dynamic, highly interconnected environments. 
Routing Domains may be recursively subdivided into Subdomains in order to reduce routing complexity. The details of a subdomain may be largely hidden from other subdomains with an attendant reduction in the volume of routing information exchanged.

Intra-Administrative Domain routing is concerned with interconnecting multiple Routing Domains within an administration. Issues may include address administration, cost recovery, and policy concerns. A moderate level of trust is assumed. The nature of the interactions between Routing Domains can range from being tightly coupled (best path routing between two RDs running different routing protocols) to being more policy-based. However, inter-RD routing within an Administrative Domain is tightly coordinated and represents a unified technical plan.

Inter-Administrative Domain routing is concerned with managing and controlling the flow of information in a highly structured way between organizations that may require formal multilateral agreements. The issues of concern at this level tend to be administrative in nature (legal/political constraints, security, access control, etc.). Multiple agreements between multiple administrations are unlikely to be implicitly transitive. This makes the analysis of policy interactions very important.

7) Mapping the $\mathrm{AD} / \mathrm{RD}$ Model Onto the Internet

The national network backbones (NSFNET, ARPANET, MILNET, NSN, and soon ESNET) can be viewed as Common Domains. Each may have sufficiently global routing knowledge to determine a path to any Internet address.

Regional networks are clearly Administrative Domains. Multilateral policy agreements are defined between the regional networks and the backbones. On the other hand, regional networks very often are tightly coupled to individual networks and campus networks in terms of routing. In this sense, a regional network could be viewed as a Routing Domain with individual campuses thought of as Subdomains.

From the standpoint of routing functionality, it is most useful to view a "classic" Autonomous system as a congruent Routing Domain and Administrative Domain. An AS as defined represents both a single IGP and a point of policy administration. The sixteen bit value now known as the Autonomous system number may instead be viewed as an Administrative Domain number.

In reality, however, many so-called Autonomous systems today do not adhere to the strict definition of an AS. In theory, an Autonomous System is quite similar to a Routing Domain, in which a high level of 
trust is made between systems, a consistent IGP is run, and full routing information is distributed. On the other hand, AS numbers have become an abstraction for policy groupings to backbones. Indeed, entire regional networks are viewed by the backbones as a single Autonomous system, even though they are not nearly as homogeneous as the AS model specifies. Such entities can be viewed as an Administrative Domain containing several Routing Domains.

Although it is true that, in this interpretation, multiple nontechnical administrations are represented within a single Administrative Domain (in conflict with the definition of an Administrative Domain), such structures require a single approach to internal routing. Even if there is not a true administration representing the collection of domains (such as a consortium), there typically is a technical committee to settle common technical issues.

8) The $\mathrm{AD} / \mathrm{RD}$ Model as an Engineering Tool

Current Autonomous Systems cross administrative boundaries with impunity. This works as long as the individual administrations operating within the common AS agree to a common technical policy for routing and network management. Connections with other backbones, regional networks, and campus networks must be planned, implemented, and managed in a coordinated fashion.

This coordination becomes more difficult, but more necessary, as the AS grows. As connectivity and policy become more complex, current Autonomous systems start to fragment. An example of this is a network that is currently a member of an NSFNET regional network but will be adding a connection to ESNET. The administrators of the network and the regional network must carefully coordinate the changes necessary to implement this connection, including possibly altering the boundaries of policy and routing. A lack of coordination could result in routing loops and policy violations.

A point that is being increasingly realized is that the entity responsible for exterior or policy routing (be it an Autonomous System or an Administrative Domain) must have a common technical policy for routing. The effects of attempting different approaches to policy and external routing while maintaining a single AS have been painfully evident in real instances in the Internet.

Under the AD/RD model, a routing domain cannot be in two Administrative Domains. For example, if a campus network wants to set its own routing policy and enforce it via management of their routers, the campus has elected to become a separate Administrative Domain. If that campus uses a common IGP with other campuses, it represents an attempt to split a Routing Domain (the regional network 
with a common IGP) across multiple Administrative Domains (the campus and the rest of the regional). Such arrangements represent dubious engineering practice, cause real routing problems, and are disallowed by the $\mathrm{AD} / \mathrm{RD}$ model.

Under the strict Autonomous system model, only one IGP can exist within an AS. However, many regional networks are successfully using multiple IGPs. The AD/RD model allows this valuable routing topology. Such a topology would also be allowed by the AS model if it were to be broadened to allow multiple IGPs, in which case an AS and an $\mathrm{AD}$ would effectively become equivalent.

9) The $\mathrm{AD} / \mathrm{RD}$ Model in a Dual Protocol Internet

As the OSI protocol suite is deployed and an OSI Internet is constructed, it is very likely that significant portions of the current TCP/IP Internet will also carry OSI traffic. Many router vendors provide dual protocol capability today, or will in the near future, and the investment in network infrastructure is such that it is unlikely that a separate, parallel internet will be established for OSI traffic.

It is logical to assume that, in many cases, the same technical and administrative boundaries will apply to both DoD IP and OSI protocols, and in some cases a single routing protocol may be used to support both protocol suites.

Thus, it would be most advantageous to have a common model and common nomenclature in order to provide a more unified, manageable routing environment. Given that the OSI Routeing Framework represents the model on which OSI routing is built, the use of the AD/RD model to describe the existing Internet is an appropriate step toward describing and building the combined internet.

10) Conclusions

The AD/RD model of routing describes the current Internet better than existing models because it describes:

- How Intra-Domain and Inter-Domain relationships work at both routing and policy level

- How routing domains and administrative domains can be hierarchically related

- The existence of multiple national peers

- A common model for dual protocol internets 
The expanding Internet has grown from the "core" model with several small attached networks to a highly interconnected environment that spans several continents. Several national peer networks serve an ever-growing set of regional networks. The AD/RD model can help Internet protocol designers abstract the functional pieces from the large Internet.

The Internet grows daily. Any model of Internet routing needs to provide a way to understand and order the growth. The ISO Routeing Framework provides a structure to handle such growth.

\section{1) References}

[1] ISO, "OSI Routeing Framework", ISO/TR 9575, 1989.

[2] Rosen, E., "Exterior Gateway Protocol", RFC 827, Bolt Beranek and Newman, October 1982 .

[3] Mills, D., "Autonomous Confederations", RFC 975, M/A COM Linkabit, February 1986.

[4] ISO, "Open Systems Interconnection--Basic Reference Model", ISO 7498 .

[5] ISO, "Internal Organization of the Network Layer", ISO 8648.

ISO documents can be obtained from the following source:

American National Standards Institute

1430 Broadway

New York, NY 10018

(212) 642-4900

Additionally, a number of private firms are authorized to distribute ISO documents.

Security Considerations

Security issues are not addressed in this memo.

Authors' Addresses

Susan Hares

Merit/NSFNET

1075 Beal Ave.

Ann Arbor, MI 48109 
Phone: (313) 936-3000

Email: skh@merit.edu

Dave Katz

Merit/NSFNET

1075 Beal Ave.

Ann Arbor, MI 48109

Phone: (313) 763-4898

Email: dkatz@merit.edu 